\title{
OPEN Deletion of CD2-like gene from the genome of African swine fever virus strain Georgia does not attenuate virulence in swine
}

\author{
Manuel V. Borca ${ }^{1 *}$, Vivian O'Donnell ${ }^{1,2}$, Lauren G. Holinka ${ }^{1}$, Guillermo R. Risatti ${ }^{1,3}$, \\ Elizabeth Ramirez-Medina ${ }^{1,3}$, Elizabeth A. Vuono ${ }^{4}$, Jishu Shi ${ }^{6}$, Sarah Pruitt ${ }^{1,5}$, Ayushi Rai ${ }^{1,5}$, \\ Ediane Silva ${ }^{1,6}$, Lauro Velazquez-Salinas ${ }^{1,6}$ \& Douglas P. Gladue $\mathbb{B}^{1 *}$ \\ The CD2-like African swine fever virus (ASFV) gene 8DR, (also known as EP402R) encodes for a \\ structural transmembrane glycoprotein that has been shown to mediate hemadsorption and be \\ involved in host immunomodulation as well as the induction of protective immune response. In \\ addition, several natural ASFV isolates showing decreased virulence in swine has been shown to be \\ non-hemadsorbing suggesting an association between altered or deleted forms of 8DR and virus \\ attenuation. Here we demonstrate that deletion of 8DR gene from the genome of ASFV Georgia2010 \\ isolate (ASFV-G- $\Delta 8 D R$ ) does not significantly alter the virulence of the virus. ASFV-G- $\Delta 8 D R$ inoculated \\ intramuscularly or intranasally (in a range of $10^{2}$ to $10^{4} \mathrm{TCID}_{50}$ ) produced a clinical disease in domestic \\ pigs indistinguishable from that induced by the same doses of the virulent parental ASFV Georgia2010 \\ isolate. In addition, viremia values in ASFV-G- $\triangle 8$ DR do not differ from those detected in animals \\ infected with parental virus. Therefore, deletion of $8 D R$ gene is not associated with a noticeable \\ decrease in virulence of the ASFV Georgia isolate.
}

African swine fever virus (ASFV) a large double stranded DNA virus and etiological agent that causes African swine fever (ASF). Depending on the isolate, swine infected with ASFV can have disease that ranges from sub-clinical to disease in which can be highly lethal ${ }^{1}$. ASF has been endemic in several sub-Saharan African countries and also in Sardinia (Italy). Recently, outbreaks of ASF have occurred in Eastern Europe and south-east Asia. These outbreaks have all originated with from an outbreak in the Caucasus region that occurred in $2007^{2}$. The isolate that is involved in the current outbreak called Georgia/2007 is highly contagious and lethal in domestic pigs. This high rate of lethality has the potential to cause large losses of domestic pigs ${ }^{3}$.

There are no vaccines currently available to prevent ASF (1). Development of experimental live-attenuated ASF vaccines, have mainly relied on the production of recombinant field isolates by genetic manipulation, in which one or more genes have been deleted from the field isolate ${ }^{4-6}$. Therefore, understanding the role of viral genes in virulence and their possible manipulation to develop attenuated virus strains to produce experimental vaccines is a critical issue.

Since its original description, the CD2-like African swine fever virus (ASFV) gene (8DR) has been the focus of intense research. $8 \mathrm{DR}$ protein has been shown to be responsible for mediating hemadsorption ${ }^{7,8}$, being critical in virus replication in tick cells ${ }^{9}$, immunomodulate the host response $\mathrm{e}^{10}$, and is actively involved in the induction of protective immune response $\mathrm{e}^{11,12}$.

\footnotetext{
${ }^{1}$ Agricultural Research Service (ARS), Plum Island Animal Disease Center, Greenport, NY, 11944, USA. ${ }^{2}$ Present address: Animal and Plant Health Inspection Service (APHIS), Plum Island Animal Disease Center, Greenport, NY, 11944, USA. ${ }^{3}$ Department of Pathobiology and Veterinary Science, University of Connecticut, Storrs, CT, 06269, USA. ${ }^{4}$ Department of Pathology and Population Medicine, Mississippi State University, P.O. Box: 6100, Mississippi State, MS, 39762, USA. ${ }^{5}$ Oak Ridge Institute for Science and Education (ORISE), Oak Ridge, TN, 37830, USA. ${ }^{6}$ Department of Anatomy and Physiology, Kansas State University, Manhattan, KS, 66506, USA. *email: Manuel.Borca@usda. gov; Douglas.Gladue@usda.gov
} 


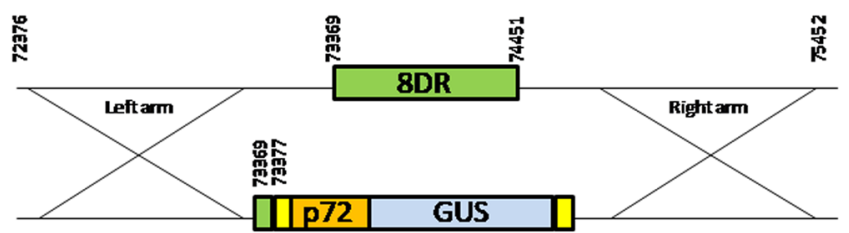

Figure 1. Diagram indicating the genomic changed performed on ASFV Georgia genome in order to develop ASFV-G- $\triangle 8 \mathrm{DR}$. The positions of the flanking arms for homologous recombination are indicated. In green is gene $8 \mathrm{DR}$ or the residual nine nucleotides of $8 \mathrm{DR}$ in the recombinant virus. Yellow boxes indicate the location of LoxP recombination sites. The p72 promoter indicated in orange and GUS is indicated in blue directly replaces the 8DR ORF.

In addition, several natural ASFV isolates showing decreased virulence in domestic swine have been shown to be non-hemadsorbing suggesting an association between altered or deleted forms of the 8DR gene and virus attenuation $^{13-15}$.

The non-pathogenic isolate from Portugal, OURT88/3 $3^{14}$, has interruptions in the 8DR ORF that encode for the CD2-like gene ${ }^{16}$ containing frameshift mutations close to the amino terminus that introduce in-frame stop codons. In addition, an alternative methionine codon appears downstream the native start codon and an additional frameshift mutation in the sequence encoding the cytoplasmic domain results in the final 215 residues not being translated. Recently, a derivative of the ASFV Georgia isolate, an attenuated field isolated Latvia isolate Lv17/WB/Rie1 was shown to be non-HA. Analysis of the 8DR gene showed a single nucleotide deletion that generates a truncated protein ${ }^{15}$. Another Portuguese attenuated non-HA isolate, NH/P68, presents several separate nucleotide deletions creating a stop codon after the first 21 residues ${ }^{17}$. We previously demonstrated that deletion of 8DR gene from highly virulent ASFV Malawi isolate ${ }^{10}$ does not significantly alter the virus virulence in terms of clinical presentation of the disease.

Here we demonstrate that deletion of 8DR gene from the genome of ASFV Georgia2010 isolate (ASFV-G- $\triangle 8 \mathrm{DR}$ ) does not significantly alter virus virulence in domestic pigs. ASFV-G- $\triangle 8 \mathrm{DR}$, inoculated intramuscularly or intranasally (in a range of $10^{2}$ to $10^{4} \mathrm{TCID}_{50}$ ), produced a clinical disease in domestic pigs comparable to that induced by similar doses of the virulent parental ASFV Georgia2010 isolate. In addition, viremia values in ASFV-G- $\triangle 8$ DR do not radically differ from those detected in animals infected with parental virus. Therefore, deletion of $8 \mathrm{DR}$ gene is not associated with decreased virulence in the ASFV Georgia isolate.

\section{Results and Discussion}

Development of an 8DR deletion mutant in ASFV-G. The role of ASFV CD2-like protein in cell culture and for ASFV virulence in swine, a deletion virus that lacks the 8DR gene was designed. 8DR was deleted by replacing nucleotides $10-1083$ of the 8 DR coding region with p72GUS using methodologies that rely on homologous recombination. The resulting recombinant virus, ASFV-G- $\triangle 8 \mathrm{DR}$, was obtained using the highly pathogenic ASFV isolate from Georgia in 2010 (ASFV-G). ASFV-G- $\triangle 8$ DR contains a deletion of 1074-bp (nucleotide positions 73,377-74,452) from ASFV-G virus. This area was replaced with a 2392-bp cassette containing the p72GUS reporter with flanking LoxP sites (more detail provided in Material and Methods) (Fig. 1). After nine passages the recombinant virus was purified. Purification was done using successive limiting dilution events using primary swine macrophage cell cultures. Once purified a virus stock was produced using primary swine macrophages with the recombinant virus ASFV-G- $\triangle 8 \mathrm{DR}$.

In order to evaluate the deletion occurred in the right location and there were no other mutations that occurred in the ASFV-G- $\Delta 8$ DR genome next generation sequencing (NGS)was used. The genome analysis confirmed the accuracy of the introduced modifications, the absence of any additional mutations and also confirmed the absence of parental ASFV-G genome in the ASFV-G- $\triangle 8 \mathrm{DR}$ stock as there were no sequencing reads that aligned to the 8DR gene as Supplementary Fig. S1. The complete sequence of ASFV-G- $\Delta 8 D$ R is included as Supplementary Fig. S2.

Primary swine macrophage replication of ASFV-G- $\Delta$ 8DR. Using primary swine macrophages the role of the $8 \mathrm{DR}$ gene in virus replication the in vitro growth characteristics was assessed by comparing ASFV-G- $\triangle 8$ DR to parental ASFV-G in a multistep growth curve. Using an MOI of 0.01 cell cultures were infected with the indicated virus and timepoints where samples were collected were at 2, 24, 48, 72 and 96 hours post-infection (hpi). When compared to parental ASFV-G virus ASFV-G- $\Delta 8$ DR displayed a slightly decreased growth kinetic (Fig. 2A). Therefore, the ability of the recombinant virus lacking 8DR does not significantly affect ASFV replication in primary swine macrophage cultures. As expected, ASFV-G- $\triangle 8 D R$ lacks the ability to form rosettes in the presence of swine red cells (Fig. 2B).

Assessment of ASFV-G- $\Delta$ 8DR virulence in swine. Several groups of 80-90 pound pigs were inoculated either intranasally (IN) or intramuscularly (IM) with a variety of doses of ASFV-G- $\triangle 8 D R$ or ASFV-G to evaluate the effect that deletion of 8DR has in ASFV-G virulence.

The groups of pigs inoculated were as follows: The first group was inoculated with $10^{2} \mathrm{HAD}_{50}$ of ASFV-G- $\triangle 8 \mathrm{DR}$. A second group of pigs was inoculated with $10^{3} \mathrm{HAD}_{50}$ of ASFV-G- $\triangle 8 \mathrm{DR}$. In comparison two other groups were inoculated with either $10^{2} \mathrm{HAD}_{50}$ or $10^{3} \mathrm{HAD}_{50}$ of ASFV-G. As expected, pigs in infected with either dose of ASFV-G an increased body temperature $\left(>40^{\circ} \mathrm{C}\right)$ was observed by day 3 or 4 post-infection (pi). 
A.

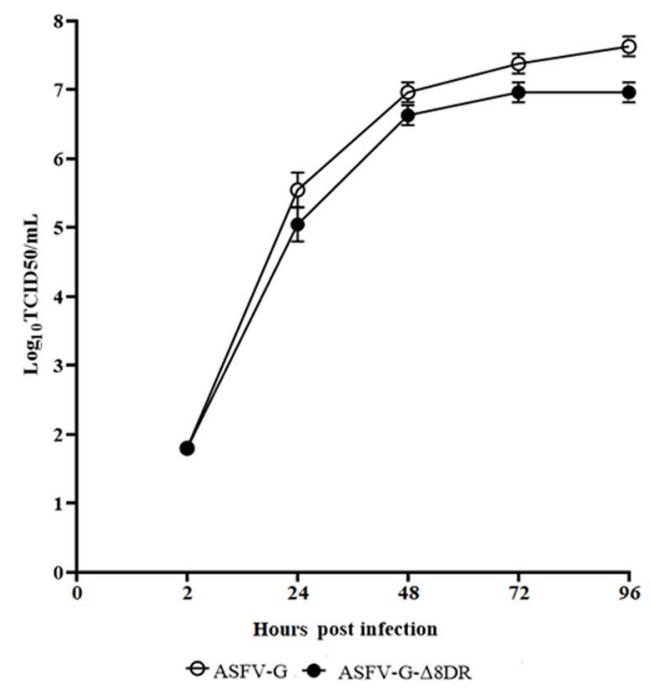

B.

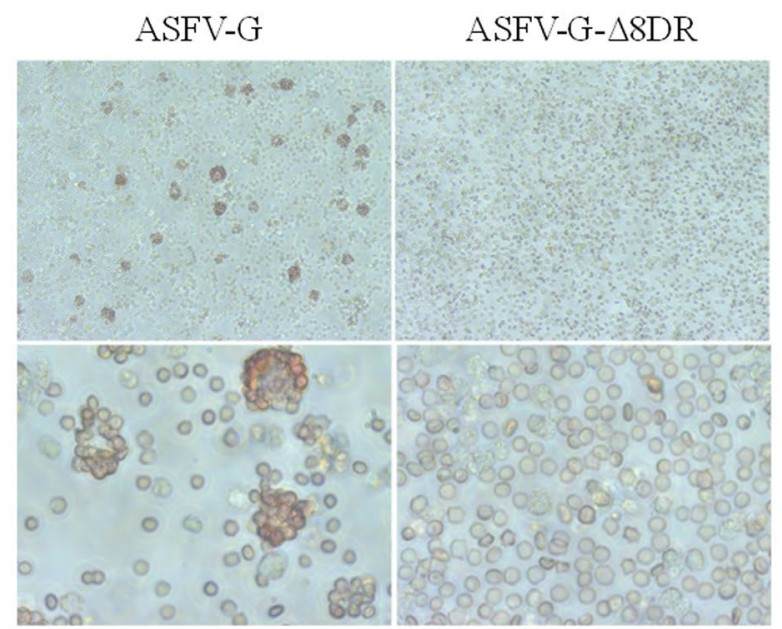

Figure 2. (A) In vitro growth characteristics of ASFV-G- $\triangle 8 D R$ and parental ASFV-G. Primary swine macrophage cell cultures were infected $(\mathrm{MOI}=0.01)$ with each of the viruses and virus yield titrated at the indicated times post-infection. Data represent means from three independent experiments. Sensitivity of virus detection: $\geq 1.8 \log _{10} \mathrm{TCID}_{50} / \mathrm{ml}$. (B) Lack of hemadsorbing activity in ASFV-G- $\triangle 8 \mathrm{DR}$ infected cells. Primary swine macrophage cell cultures were infected $(\mathrm{MOI}=10)$ with ASFV-G- $\Delta 8 \mathrm{DR}$ or parental ASFV-G infected in the presence of swine red cells.

This increase in body temperature was then followed by the clinical appearance of signs associated with ASF including purple skin discoloration, anorexia, diarrhea, depression, and staggering gait. Over time the signs of the disease aggravated progressively and animals were euthanized in extremis by approximately day 7 pi. No marked differences were observed in the kinetics of clinical signs presentation or lethality between animals inoculated with either $10^{2}$ or $10^{4} \mathrm{TCID}_{50}$ of ASFV-G (Fig. 3A,C). Interestingly, animals inoculated with $10^{2}$ or $10^{4} \mathrm{TCID}_{50}$ of ASFV-G- $\triangle 8$ DR did not drastically differed from those inoculated with similar doses of ASFV-G (Table 1). Appearance of clinical signs associated with the disease almost overlaps with that of the animals inoculated with ASFV-G as well as the evolution of disease severity and time of death. As in the case of ASFV-G, no clear differences were observable between groups receiving different doses of ASFV-G- $\Delta 8 \mathrm{DR}$.

Viremia titers of animals inoculated IM with different doses of ASFV-G- $\Delta 8$ DR or ASFV-G showed differences, being titers of animals infected with ASFV-G approximately 100 fold higher than those of animals infected with ASFV-G- $\triangle 8 \mathrm{DR}$ in samples taken both at 4- and 7-days post infection (dpi) (Fig. 4A).

Next, we attempted to improve the possibilities of showing any effect of 8 DR deletion on virus virulence by using a less stringent system, the IN route. One group was inoculated with $10^{3} \mathrm{TCID}_{50}$ of ASFV-G- $\Delta 8 \mathrm{DR}$, a second group was inoculated with $10^{4} \mathrm{TCID}_{50}$ of ASFV-G- $\triangle 8 \mathrm{DR}$, while a third and a fourth groups were inoculated with $10^{3}$ and $10^{4} \mathrm{TCID}_{50}$ of ASFV-G, respectively. In both groups infected with ASFV-G animals exhibited the outcome of the disease with increased body temperature $\left(>40^{\circ} \mathrm{C}\right)$ by day 4 or 5 pi followed by the full appearance of clinical disease (Table 2 and Fig. 3B,D), which aggravated progressively over time and animals were euthanized in extremis by day $9-10$ pi. This result indicates that IN inoculation of similar doses of ASFV-G truly represent a 


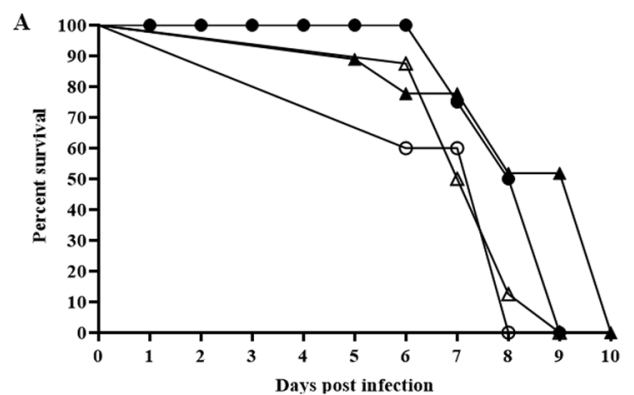

$\theta$ ASFV-G $10^{2} \bullet$ ASFV-G- $\triangle 8$ DR $10^{2} \triangle$ ASFV-G $10^{4} \star$ ASFV-G- $\triangle 8$ DR $10^{4}$

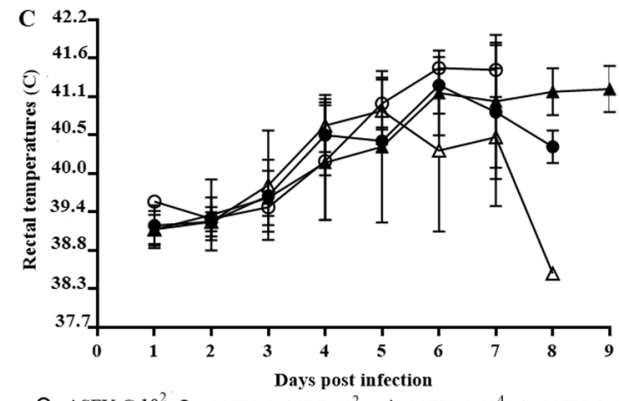

$\ominus$ ASFV-G $10^{2}$

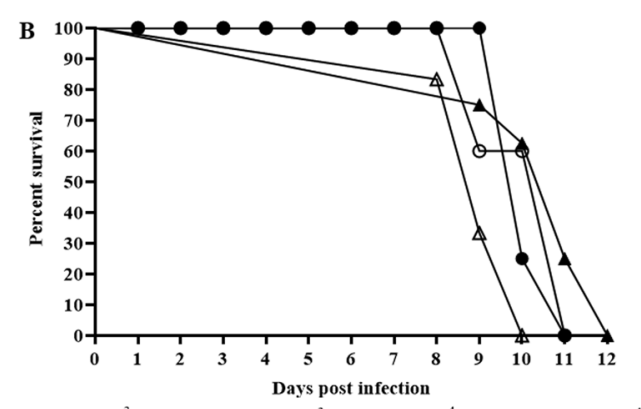

$\theta$ ASFV-G $10^{3}-$ ASFV-G- $\triangle 8$ DR $10^{3} ₫$ ASFV-G $10^{4} \star$ ASFV-G- $\triangle 8$ DR $10^{4}$

Figure 3. Kinetics of lethality $(\mathbf{A}, \mathbf{B})$ and body temperature $(\mathbf{C}, \mathbf{D})$ in pigs inoculated $\mathrm{IM}\left(10^{2}\right.$ or $\left.10^{4} \mathrm{TCID}_{50}\right)$ $(\mathbf{A}, \mathbf{C})$ or IN $\left(10^{3}\right.$ or $10^{4}$ TCID $\left._{50}\right)(\mathbf{B}, \mathbf{B})$ with either ASFV-G- $\triangle 8 \mathrm{DR}$ (filled symbols), or of ASFV-G (empty symbols). Body temperature data is shown as average values and their $\mathrm{SD}$ in each of the group.

\begin{tabular}{|l|l|l|l|l|l|}
\hline & & & \multicolumn{2}{l|}{ Fever } \\
\cline { 3 - 6 } Virus & $\begin{array}{l}\text { No. of survivors/ } \\
\text { total }\end{array}$ & $\begin{array}{l}\text { Mean time to death } \\
(\text { days } \pm \text { SD) }\end{array}$ & $\begin{array}{l}\text { No. of days to onset } \\
(\text { days } \pm \text { SD }\end{array}$ & $\begin{array}{l}\text { Duration No. of days } \\
(\text { days } \pm \text { SD) }\end{array}$ & $\begin{array}{l}\text { Maximum daily } \\
\text { temp }\left({ }^{\circ} \mathbf{C} \pm \text { SD) }\right.\end{array}$ \\
\hline ASFV-G $10^{2} \mathrm{TCID}_{50}$ & $0 / 4$ & $7(1.41)^{(1)}$ & $4.5(0.71)$ & $2.5(0.71)$ & $41.4(0.04)$ \\
\hline ASFV-G $10^{4} \mathrm{TCID}_{50}$ & $0 / 8$ & $7.5(0.92)$ & $3.63(0.92)$ & $3.88(0.84)$ & $40.9(0.48)$ \\
\hline ASFV-G- $\Delta 8 \mathrm{DR} 10^{2} \mathrm{TCID}_{50}$ & $0 / 4$ & $8.25(0.96)$ & $3.75(0.5)$ & $4.5(0.58)$ & $41.28(0.41)$ \\
\hline ASFV-G- $\Delta 8 \mathrm{R} 10^{4} \mathrm{TCID}_{50}$ & $0 / 8$ & $8.14(2.04)$ & $4.71(1.25)$ & $3.43(1.51)$ & $41.17(0.61)$ \\
\hline
\end{tabular}

Table 1. Swine survival and fever response following intramuscular infection with different doses of ASFVG- $\triangle 8$ DR or parental ASFV-G. ${ }^{(1)}$ All animals were euthanized due to humanitarian reasons following the corresponding IACUC protocol.

less stringent infectious model since time of death was extended in at least 2 days when compared to that in animals infected by the IM route (Table 1 and Fig. 3A,C). Interestingly, animals infected either with $10^{3}$ or $10^{4} \mathrm{TCID}_{50}$ of ASFV-G- $\triangle 8$ DR presented, again, a disease kinetics similar as animals that were inoculated with ASFV-G. Both the time of outcome of clinical disease and the evolution of severity of the clinical signs related with the disease were very similar to those present in animals inoculated with the ASFV-G (Table 2 and Fig. 3B,D).

Comparison of viremia titers of animals inoculated IN with either ASFV-G- $\triangle 8$ DR or ASFV-G showed some differences, particularly when the 4-dpi sampling point was considered. Animals infected with ASFV-G presented viremia titers approximately 1,000 folds higher than those of animals infected with ASFV-G- $\Delta 8 \mathrm{DR}$ at 4 dpi, while the differences were reduced by only 10 fold by 7 dpi (Fig. 4B).

Therefore, the results presented here clearly demonstrated that deletion of the 8DR gene from the genome of the highly virulent ASFV-G isolate does not significantly alter virus virulence in swine. By using two different doses of virus and two different routes of inoculation no clear differences were observed in the onset of the disease, the severity of the clinical signs, the evolution of the clinical disease or the time of death (or humanitarian euthanasia) between animals infected with ASFV-G- $\triangle 8 D$ D or the parental ASFV-G. Analysis of virological data indicate that viremia values are decreased in ASFV-G- $\triangle 8 \mathrm{DR}$ infected animals when compared to those infected with ASFV-G. These results suggest there is no a direct association between the concentration of virus circulating and the severity and evolution of clinical disease.

Overall, the results reported here would indicate that disruption of 8DR gene function in the Georgia strain would not lead to virus attenuation as it might had happened with some naturally attenuated field isolates. Interestingly, differential effects on virus virulence by the deletion of a particular gene, depending of the ASFV strain considered, has been already described in ASFV. For instance, deletion of the NL gene ${ }^{18-20}$, UK gene U $^{19,21,22}$ and $9 \mathrm{GL}^{6,21}$ gene have been already described to differentially affect virus virulence regarding the ASFV isolate analyzed, opening the possibility that also deletion of the CD2 like gene may have a different effect depending of 


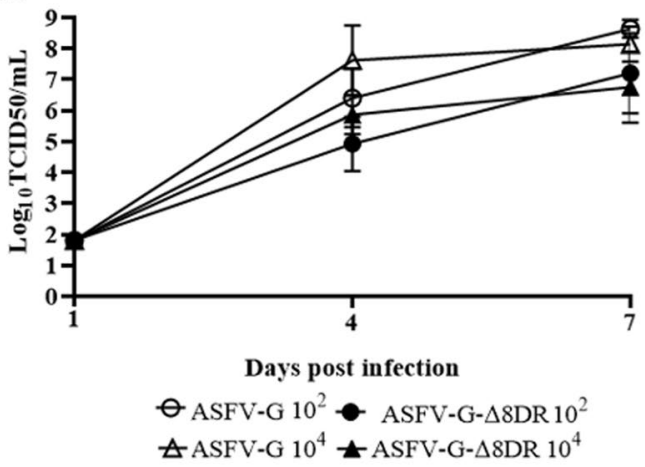

B

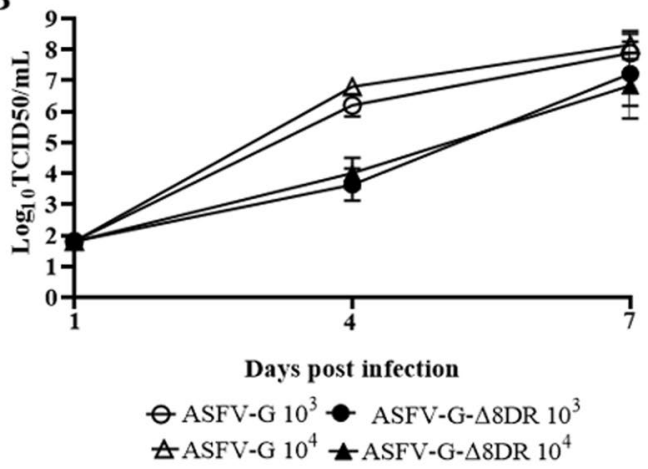

Figure 4. Viremia titers detected in pigs inoculated IM $\left(10^{2}\right.$ or $\left.10^{4} \mathrm{TCID}_{50}\right)$ or IN $\left(10^{3}\right.$ or $\left.10^{4} \mathrm{TCID}_{50}\right)$ with either ASFV-G- $\triangle 8$ DR (filled symbols), or of ASFV-G (empty symbols). Viremia values is shown as average values and their SD in each of the group. Sensitivity of virus detection: $\geq \log _{10} 1.8 \log _{10} \mathrm{TCID}_{50} / \mathrm{ml}$.

\begin{tabular}{|c|c|c|c|c|c|}
\hline \multirow[b]{2}{*}{ Virus } & \multirow[b]{2}{*}{$\begin{array}{l}\text { No. of } \\
\text { survivors/total }\end{array}$} & \multirow[b]{2}{*}{$\begin{array}{l}\text { Mean time to death } \\
\text { (days } \pm \text { SD) }\end{array}$} & \multicolumn{3}{|l|}{ Fever } \\
\hline & & & $\begin{array}{l}\text { No. of days to onset } \\
\text { (days } \pm \text { SD }\end{array}$ & $\begin{array}{l}\text { Duration No. of days } \\
\text { (days } \pm \text { SD) }\end{array}$ & $\begin{array}{l}\text { Maximum daily } \\
\text { temp }\left({ }^{\circ} \mathrm{C} \pm \mathrm{SD}\right)\end{array}$ \\
\hline ASFV-G $10^{3} \mathrm{TCID}_{50}$ & $0 / 4$ & $10(1.15)^{(1)}$ & $5.25(0.96)$ & $4.75(1.17)$ & $41.5(0.28)$ \\
\hline ASFV-G $10^{4} \mathrm{TCID}_{50}$ & $0 / 8$ & $9.17(0.75)$ & $4(0)$ & $5.17(0.75)$ & $41.3(0.92)$ \\
\hline ASFV-G- $\Delta 8 \mathrm{DR} 10^{3} \mathrm{TCID}_{50}$ & $0 / 4$ & $10.25(0.5)$ & $5.75(0.5)$ & $4.5(0.58)$ & $41.3(0.19)$ \\
\hline ASFV-G- $\Delta 8$ DR $10^{4}$ TCID $_{50}$ & $0 / 6$ & $10.63(1.19)$ & $(0.54)$ & $4.12(1.36)$ & $41.2(0.38)$ \\
\hline
\end{tabular}

Table 2. Swine survival and fever response following intranasal infection with different doses of ASFVG- $\triangle 8$ DR or parental ASFV-G. ${ }^{(1)}$ All animals were euthanized due to humanitarian reasons following the corresponding IACUC protocol.

the ASFV isolate used for the study. Attenuation was reported by deleting the CD2-like gene in ASFV Ba71 this ${ }^{23}$ supports the concept that deletion of a specific gene may have different effect in the virus phenotype depending in part of the genetic background where the deletion is performed. Importantly, results reported here closely concur with information reported for the highly virulent ASFV Malawi isolate (10) where, although significant decreased virus titers were detected in blood and organs of animals infected with a recombinant virus harboring a deletion of the $8 \mathrm{DR}$ gene, no differences in the characteristic of clinical presentation and lethality were found when compared with animals infected with the parental virulent virus. It should be noted that in both cases, no additional genetic modifications were accidentally introduced in the recombinant viruses indicating a full genome integrity besides the deletion of the 8DR gene.

The fact that several natural attenuated field isolates cannot mediate HA should be cautiously considered in terms of associating a disruption or deletion of the CD2 like gene and virus attenuation. Naturally occurring avirulent non-hemadsorbing viruses, like OURT 88/3 ${ }^{14}$, NH/P68 (NHV) (17) and Latvia 2017 (15) harbor mutations in $8 D R$ that lead to lack of production or the production of a non-functional CD2 homologue proteins. However, both, OURT 88/3 and NH/P68 (NHV), also bear deletions of several MGF 360 and 505 genes that most likely are a major cause leading to attenuation. The full-genome sequence of Latvia 2017 is still not available limiting the analysis of the MGF 360 and 505 loci. Therefore, available data suggest that MGF 360 or 505 are necessary for ASFV virulence (5) but not $8 \mathrm{DR}$, pointing to the conclusion that the CD2 homologue may play no significant role in the loss of virus virulence in those naturally attenuated field isolates.

\section{Materials and Methods}

Cell cultures and viruses. Primary swine macrophage cell cultures were prepared from defibrinated swine blood as previously described ${ }^{20,24}$. Briefly, heparin-treated swine blood was incubated at $37^{\circ} \mathrm{C}$ for 1 hour to separate the erythrocyte fraction. Plasma was overlaid over Ficoll-Paque specific gravity, 1.077 gradient (GE Healthcare Life Sciences, Marlborough, MA). The monocyte/macrophage cell fraction was cultured with RPMI 1640 Medium (Life Technologies, Grand Island, NY) with 30\% L929 supernatant and 20\% fetal bovine serum (HI-FBS, Thermo Scientific, Waltham, MA). Adherent cells were detached 24hrs later using $10 \mathrm{mM}$ EDTA in phosphate buffered saline (PBS) and were then reseeded into 6- or 96-well dishes at a density of $5 \times 10^{6}$ cells per $\mathrm{ml}$ for use in assays 24 hours later.

ASFV Georgia (ASFV-G) was a field isolate kindly provided by Dr. Nino Vepkhvadze, from the Laboratory of the Ministry of Agriculture (LMA) in Tbilisi, Republic of Georgia ${ }^{25}$.

Comparative growth curves were done as previously described ${ }^{26,27}$. between ASFV-G- $\Delta 8 \mathrm{DR}$ and parental ASFV-G were performed in primary swine macrophage cell cultures. Preformed monolayers were prepared in 24-well plates and infected at a MOI of 0.01 (based on $\mathrm{HAD}_{50}$ and $\mathrm{TCID}_{50}$ previously determined in primary swine macrophage cell cultures, ASFV-G had identical values for $\mathrm{HAD}_{50}$ and $\mathrm{TCID}_{50}$. ASFV-G- $\triangle 8 \mathrm{DR}$ was 
titrated using $\mathrm{TCID}_{50}$ due to the inability to measure the $\mathrm{HAD}_{50}$. After 1 hour of adsorption at $37^{\circ} \mathrm{C}$ under $5 \%$ $\mathrm{CO}_{2}$ the inoculum was removed, and the cells were rinsed two times with PBS. The monolayers were then rinsed with macrophage media and incubated for $2,24,48,72$ and 96 hours at $37^{\circ} \mathrm{C}$ under $5 \% \mathrm{CO}_{2}$. At appropriate times post-infection, the cells were frozen at $\leq-70^{\circ} \mathrm{C}$ and the thawed lysates were used to determine titers by $\mathrm{TCID}_{50} / \mathrm{ml}$ in primary swine macrophage cell cultures. All samples were run simultaneously to avoid inter-assay variability.

Virus titration using macrophage cell cultures were performed in 96-well plates, diluting virus in macrophage medium as previously described ${ }^{27}$. Presence of virus was assessed by the detection of cytopathic effect in the infected cells. Virus titers were calculated as tissue culture infectious doses $\left(\mathrm{TCID}_{50} / \mathrm{ml}\right)$ as previously described ${ }^{28}$.

Construction of the recombinant viruses. Recombinant ASFV-G- $\Delta 8 \mathrm{DR}$ was generated by homologous recombination between the parental ASFV genome and a recombination transfer vector following infection and transfection of swine macrophage cell cultures ${ }^{20,29}$. The recombinant transfer vector (p72GUS $\Delta 8 \mathrm{DR}$ ) contains a left recombination arm that is $1000 \mathrm{bp}$ upstream of ORF 8DR identical to ASFV-G nucleotide positions 72,37673,376 , followed by a LoxP recombination site the $\mathrm{p} 72$ promoter identical to ASFV-G nucleotide positions on the negative strand 105720-105533, followed by -glucuronidase gene (GUS), followed by a second LoxP recombination site followed by a right recombination arm that is $1000 \mathrm{bp}$ downstream of 8-DR identical to ASFV-G nucleotide positions 74,452-75,452 (Fig. 2). Recombinant transfer vectors p72GUS $\Delta$ 8DR was obtained by DNA synthesis (Epoch Life Sciences, Sugar Land, TX, USA). Macrophage cell cultures were infected with ASFV-G and transfected with p72GUS $\Delta 8$ DR. Recombinant ASFV-G- $\Delta 8$ DR was purified to homogeneity by successive rounds of limiting dilution purification.

Complete sequencing of ASFV genomes using next generation sequencing. Macrophage cells were seeded as described and infected with ASFV, once the cytopathic effect was evident throughout the monolayer, DNA was isolated as described previously from cells infected with ASFV ${ }^{25}$. The extracted DNA was then used to completely sequence the virus DNA as previously described ${ }^{25}$. In Brief, the viral DNA was sheared using enzymatic reactions assessed for the distribution of size fragmentation, then ligation of identifying barcodes using an adapter sequence were added to the DNA fragments. Using a Pippin Prep ${ }^{\mathrm{TM}}$ (Sage Science, Beverly, MA) the required size range of the library was collected and normalized. We then used this DNA library for NGS sequencing using an Ion Torrent following the manufactures protocol. Sequence analysis was performed using CLC Genomics Workbench software (CLCBio, Waltham, MA). We had a total of 2,550,135 reads in which 77,478 reads were mapped to ASFV-G, the mean read length of the mapped reads was 156.87 with a total read length of $12,154,171$. The alignment of reads in the region of $\triangle 8 \mathrm{DR}$ is included as Supplementary Fig. 1.

Ethics statement. Animal experiments were performed under biosafety level 3AG conditions in the animal facilities at Plum Island Animal Disease Center (PIADC. All experimental procedures were carried out in compliance with the Animal Welfare Act (AWA), the 2011 Guide for Care and Use of Laboratory Animals, the 2002 PHS Policy for the Humane Care and Use of Laboratory Animals, and U.S. Government Principles for Utilization and Care of Vertebrate Animals Used in Testing, Research and Training (IRAC 1985), as well as specific animal protocols reviewed and approved by the PIADC Institutional Animal Care and Use Committee of the US Departments of Agriculture and Homeland Security (protocol number 225.04-16-R, 09-07-16).

Animal experiments. ASFV-G- $\triangle 8 \mathrm{DR}$ was assessed for its virulence phenotype relative to the parental ASFV-G virus using 80-90-pound commercial breed swine similar to previous studies ${ }^{19,27}$. Five pigs were inoculated intramuscularly (IM) with either $10^{2}$ or $10^{3}$ TCID $_{50}$ of ASFV-G- $\triangle 8$ DR and compared with an additional two groups inoculated with similar doses of or ASFV-G. Clinical signs (anorexia, depression, fever, purple skin discoloration, staggering gait, diarrhea and cough) and changes in body temperature were recorded daily throughout the experiment.

Received: 30 August 2019; Accepted: 23 December 2019;

Published online: 16 January 2020

\section{References}

1. Tulman, E. R., Delhon, G. A., Ku, B. K. \& Rock, D. L. In Lesser Known Large dsDNA Viruses Vol. 328 Current Topics in Microbiology and Immunology 43-87 (Springer-Verlag Berlin Heidelberg, 2009).

2. Chapman, D. A. et al. Genomic analysis of highly virulent Georgia 2007/1 isolate of African swine fever virus. Emerging infectious diseases 17, 599-605, https://doi.org/10.3201/eid1704.101283 (2011).

3. Costard, S. et al. African swine fever: how can global spread be prevented? Philosophical transactions of the Royal Society of London. Series B, Biological sciences 364, 2683-2696, https://doi.org/10.1098/rstb.2009.0098 (2009).

4. Lewis, T. et al. An African swine fever virus ERV1-ALR homologue, 9GL, affects virion maturation and viral growth in macrophages and viral virulence in swine. Journal of virology 74, 1275-1285 (2000).

5. O’Donnell, V. et al. African Swine Fever Virus Georgia Isolate Harboring Deletions of MGF360 and MGF505 Genes Is Attenuated in Swine and Confers Protection against Challenge with Virulent Parental Virus. Journal of virology 89, 6048-6056, https://doi. org/10.1128/JVI.00554-15 (2015).

6. O’Donnell, V. et al. African Swine Fever Virus Georgia 2007 with a Deletion of Virulence-Associated Gene 9GL (B119L), when Administered at Low Doses, Leads to Virus Attenuation in Swine and Induces an Effective Protection against Homologous Challenge. Journal of virology 89, 8556-8566, https://doi.org/10.1128/JVI.00969-15 (2015).

7. Borca, M. V. et al. An African swine fever virus gene with similarity to the T-lymphocyte surface antigen CD2 mediates hemadsorption. Virology 199, 463-468, https://doi.org/10.1006/viro.1994.1146 (1994).

8. Rodriguez, J. M., Yanez, R. J., Almazan, F., Vinuela, E. \& Rodriguez, J. F. African swine fever virus encodes a CD2 homolog responsible for the adhesion of erythrocytes to infected cells. Journal of virology 67, 5312-5320 (1993). 
9. Rowlands, R. J., Duarte, M. M., Boinas, F., Hutchings, G. \& Dixon, L. K. The CD2v protein enhances African swine fever virus replication in the tick vector, Ornithodoros erraticus. Virology 393, 319-328, https://doi.org/10.1016/j.virol.2009.07.040 (2009).

10. Borca, M. V. et al. Deletion of a CD2-like gene, 8-DR, from African swine fever virus affects viral infection in domestic swine. Journal of virology 72, 2881-2889 (1998).

11. Ruiz-Gonzalvo, F., Rodriguez, F. \& Escribano, J. M. Functional and immunological properties of the baculovirus-expressed hemagglutinin of African swine fever virus. Virology 218, 285-289, https://doi.org/10.1006/viro.1996.0193 (1996).

12. Burmakina, G. et al. African swine fever virus serotype-specific proteins are significant protective antigens for African swine fever. The Journal of general virology 97, 1670-1675, https://doi.org/10.1099/jgv.0.000490 (2016).

13. Leitao, A. et al. The non-haemadsorbing African swine fever virus isolate ASFV/NH/P68 provides a model for defining the protective anti-virus immune response. The Journal of general virology 82, 513-523, https://doi.org/10.1099/0022-1317-82-3-513 (2001).

14. Boinas, F. S., Hutchings, G. H., Dixon, L. K. \& Wilkinson, P. J. Characterization of pathogenic and non-pathogenic African swine fever virus isolates from Ornithodoros erraticus inhabiting pig premises in Portugal. The Journal of general virology 85, 2177-2187, https://doi.org/10.1099/vir.0.80058-0 (2004).

15. Gallardo, C. et al. Attenuated and non-haemadsorbing (non-HAD) genotype II African swine fever virus (ASFV) isolated in Europe, Latvia 2017. Transbound Emerg Dis 66, 1399-1404, https://doi.org/10.1111/tbed.13132 (2019).

16. Chapman, D. A., Tcherepanov, V., Upton, C. \& Dixon, L. K. Comparison of the genome sequences of non-pathogenic and pathogenic African swine fever virus isolates. The Journal of general virology 89, 397-408, https://doi.org/10.1099/vir.0.83343-0 (2008).

17. Portugal, R. et al. Related strains of African swine fever virus with different virulence: genome comparison and analysis. The Journal of general virology 96, 408-419, https://doi.org/10.1099/vir.0.070508-0 (2015).

18. Afonso, C. L., Zsak, L., Carrillo, C., Borca, M. V. \& Rock, D. L. African swine fever virus NL gene is not required for virus virulence. The Journal of general virology 79(Pt 10), 2543-2547, https://doi.org/10.1099/0022-1317-79-10-2543 (1998).

19. Ramirez-Medina, E. et al. Differential Effect of the Deletion of African Swine Fever Virus Virulence-Associated Genes in the Induction of Attenuation of the Highly Virulent Georgia Strain. Viruses 11, https://doi.org/10.3390/v11070599 (2019).

20. Zsak, L., Lu, Z., Kutish, G. F., Neilan, J. G. \& Rock, D. L. An African swine fever virus virulence-associated gene NL-S with similarity to the herpes simplex virus ICP34.5 gene. Journal of virology 70, 8865-8871 (1996).

21. O'Donnell, V. et al. African swine fever virus Georgia isolate harboring deletions of 9GL and MGF360/505 genes is highly attenuated in swine but does not confer protection against parental virus challenge. Virus research 221, 8-14, https://doi.org/10.1016/j. virusres.2016.05.014 (2016)

22. Zsak, L. et al. A nonessential African swine fever virus gene UK is a significant virulence determinant in domestic swine. Journal of virology 72, 1028-1035 (1998).

23. Monteagudo, P. L. et al. BA71DeltaCD2: a New Recombinant Live Attenuated African Swine Fever Virus with Cross-Protective Capabilities. Journal of virology 91, https://doi.org/10.1128/JVI.01058-17 (2017).

24. Borca, M. V., Holinka, L. G., Berggren, K. A. \& Gladue, D. P. CRISPR-Cas9, a tool to efficiently increase the development of recombinant African swine fever viruses. Scientific reports 8, 3154, https://doi.org/10.1038/s41598-018-21575-8 (2018).

25. Krug, P. W. et al. The progressive adaptation of a georgian isolate of African swine fever virus to vero cells leads to a gradual attenuation of virulence in swine corresponding to major modifications of the viral genome. Journal of virology 89, 2324-2332, https://doi.org/10.1128/JVI.03250-14 (2015).

26. Borca, M. V. et al. The L83L ORF of African swine fever virus strain Georgia encodes for a non-essential gene that interacts with the host protein IL-1beta. Virus research 249, 116-123, https://doi.org/10.1016/j.virusres.2018.03.017 (2018).

27. Borca, M. V. et al. Development of a fluorescent ASFV strain that retains the ability to cause disease in swine. Scientific reports 7 , 46747, https://doi.org/10.1038/srep46747 (2017).

28. Reed, L. J. M. H. A simple method of estimating fifty percent endpoints. The American Journal of Hygiene 27, 493-497 (1938).

29. Neilan, J. G. et al. A conserved African swine fever virus IkappaB homolog, $5 \mathrm{EL}$, is nonessential for growth in vitro and virulence in domestic swine. Virology 235, 377-385, https://doi.org/10.1006/viro.1997.8693 (1997).

\section{Acknowledgements}

This project was funded under Award Numbers HSHQDC-11-X-00077 and HSHQPM-12-X-00005 through an interagency agreement with the Science and Technology Directorate of the U.S. Department of Homeland Security. We specially would like to thank Melanie Prarat for editing the manuscript. This research was supported in part by an appointment to the Plum Island Animal Disease Center (PIADC) Research Participation Program administered by the Oak Ridge Institute for Science and Education (ORISE) through an interagency agreement between the U.S. Department of Energy (DOE) and the U.S. Department of Agriculture (USDA). ORISE is managed by ORAU under DOE contract number DE-SC0014664. All opinions expressed in this paper are the author's and do not necessarily reflect the policies and views of USDA, ARS, APHIS, DHS, DOE, or ORAU/ ORISE.

\section{Author contributions}

The experiments were conceived by M.B., D.G., V.O., G.R. The experiments were carried out by L.H., E.R., E.V., J.S., S.P., A.R., E.S., L.V. Formal analysis was done by M.B., D.G., V.O., G.R. The manuscript was written by M.B., D.G. The manuscript was revised by M.B., D.G., V.O., G.R., L.H., E.R., E.V., J.S., S.P., A.R., E.S., L.V.

\section{Competing interests}

The authors declare no competing interests.

\section{Additional information}

Supplementary information is available for this paper at https://doi.org/10.1038/s41598-020-57455-3.

Correspondence and requests for materials should be addressed to M.V.B. or D.P.G.

Reprints and permissions information is available at www.nature.com/reprints.

Publisher's note Springer Nature remains neutral with regard to jurisdictional claims in published maps and institutional affiliations. 
(c) (i) Open Access This article is licensed under a Creative Commons Attribution 4.0 International License, which permits use, sharing, adaptation, distribution and reproduction in any medium or format, as long as you give appropriate credit to the original author(s) and the source, provide a link to the Creative Commons license, and indicate if changes were made. The images or other third party material in this article are included in the article's Creative Commons license, unless indicated otherwise in a credit line to the material. If material is not included in the article's Creative Commons license and your intended use is not permitted by statutory regulation or exceeds the permitted use, you will need to obtain permission directly from the copyright holder. To view a copy of this license, visit http://creativecommons.org/licenses/by/4.0/.

(C) The Author(s) 2020 